\title{
Preoperative Evaluation of Posthepatectomy Liver Failure Using MRI-Based Liver Function Indices in Child-Pugh Class A Patient
}

\author{
Shigeru Matsushima1*, Yozo Sato', Hidekazu Yamaura1, Mina Kato1, Yui Onoda1, \\ Shinichi Murata ${ }^{1}$, Yasuhiro Shimizu², Yasutomi Kinosada ${ }^{3}$, Hideyuki Nishiofuku4, \\ Yoshitaka Inaba ${ }^{1}$ \\ ${ }^{1}$ Department of Diagnostic and Interventional Radiology, Aichi Cancer Center Hospital, Nagoya, Japan \\ ${ }^{2}$ Department of Gastroenterological Surgery, Aichi Cancer Center Hospital, Nagoya, Japan \\ ${ }^{3}$ Departments of Biomedical Informatics, Gifu University Graduate School of Medicine, Gifu, Japan \\ ${ }^{4}$ Department of Radiology, Nara Medical University, Nara, Japan \\ Email: *smts@aichi-cc.jp
}

Received 31 May 2016; accepted 25 June 2016; published 28 June 2016

Copyright (C) 2016 by authors and Scientific Research Publishing Inc.

This work is licensed under the Creative Commons Attribution International License (CC BY).

http://creativecommons.org/licenses/by/4.0/

(c) (i) Open Access

\begin{abstract}
Purpose: To evaluate posthepatectomy liver failure (PHLF) using gadoxetic acid-enhanced magnetic resonance imaging (MRI) with a measure of relative liver enhancement (RLE) on hepatobiliary phase images, thereby facilitating safe liver resection. Methods: Twenty patients in ChildPugh class A underwent tumor excision surgery and indocyanine green (ICG) clearance of future remnant liver (FRL) (ICG-Krem) values were >0.05. PHLF was evaluated using the grading system of the International Study Group of Liver Surgery (ISGLS). The RLE value was defined as the signal gain percentage between the precontrast and hepatocellular images. In the whole liver and FRL, theRLE value measured the tumor-free liver parenchyma in RLE images. We examined the correlation between indocyanine green clearance (ICG-K) and MRI-based liver function in the whole liver. Preoperative PHLF evaluation was predicted using remnant hepatocellular uptake index (rHUI), remnant RLE (rRLE), coefficient variation of Rrle [Cv(rRLE)], and ICG-Krem corrected by heterogeneous liver function(HLF-ICG-Krem). Results: HLF-ICG-Krem and rRLE values correlated with INRs after postoperative day five ( $r=-0.55$ and $0.46, p=0.01$ and 0.04 , respectively). Furthermore, HLF-ICG-Krem values $\leq \mathbf{0 . 0 5}$ detected two patients with higher INRs after postoperative day five. On the other hand, neither rHUI nor Cv(rRLE) was correlated with INRs after postoperative
\end{abstract}

\footnotetext{
${ }^{*}$ Corresponding author.
}

How to cite this paper: Matsushima, S., Sato, Y., Yamaura, H., Kato, M., Onoda, Y., Murata, S., Shimizu, Y., Kinosada, Y., Nishiofuku, H. and Inaba, Y. (2016) Preoperative Evaluation of Posthepatectomy Liver Failure Using MRI-Based Liver Function Indices in Child-Pugh Class A Patient. Open Journal of Radiology, 6, 147-156. http://dx.doi.org/10.4236/ojrad.2016.62022 
day five ( $r=0.28$, and -0.03 , respectively; $p>0.05$ for both). HLF-ICG-Krem was significantly lower with PHLF than without PHLF ( $p=0.005)$. Conclusion: HLF-ICG-Krem is useful for evaluating PHLF more correctly.

\title{
Keywords
}

\author{
Posthepatectomy Liver Failure, Heterogeneous Liver Function, Gadoxetic Acid, Relative Liver \\ Enhancement, Indocyanine Green Clearance
}

\section{Introduction}

Preoperative evaluation of future remnant liver (FRL) function is crucial in determining whether a patient can safely undergo liver resection. One reliable index for estimating posthepatectomy liver failure (PHLF) is indocyanine green (ICG) clearance of the FRL (ICG-Krem), which is calculated as the ratio of FRL volume to total liver volume [1] [2]. The ICG-Krem threshold required for safe liver resection is $>0.05$ [3]; however, ICG-Krem assumes homogenous uptake throughout the liver.

Gadoxetic acid disodium is a developed magnetic resonance (MR) contrast agent for hepatocellular imaging. Several reports have discussed liver function assessments using MR images acquired using a variety of liverspecific contrast agents [4]-[13]. Recent reports suggested that gadoxetic acid disodiumcan also be used as a tracer in liver function testing [5] [6], and the relative liver enhancement (RLE) on hepatobiliary phase images is a potentially useful method for heterogeneous liver function (HLF) imaging by magnetic resonance imaging (MRI) [13] [14]. The objective of this study was to detect PHLF that was not detected by ICG-Krem. We corrected ICG-Krem for HLF using a coefficient variation of the FRL-RLE (rRLE) value [Cv(rRLE)]. Finally, we conducted a preoperative evaluation of PHLF using ICG-Krem while also considering HLF (HLF-ICG-Krem).

\section{Methods}

\subsection{Patients}

This prospective study was approved by the institutional review committee of our institution and all patients provided written informed consent prior to participation. From April 2013 to March 2015, 20 patients (15 men, 5 women; age range, 43 - 82 years; median age, 67 years) with HCC (8 patients) and metastatic liver tumors (12 patients) underwent gadoxetic acid disodium-enhanced MRI and surgery for tumor excision; among these eight underwent a partial hepatectomy. Of these, one patient had grade 1 fibrosis, one had grade 3 , and one had grade 4. All patients without portal hypertension had Child-Pugh class A scores [15] and ICG-Krem values > 0.05 [3]. All patients underwent prearranged surgeries without any complications. There are no exclusion criteria in addition to the above-mentioned standard in this study. PHLF was evaluated using the International Study Group of Liver Surgery (ISGLS) grading system [16].

\subsection{Biochemical Liver Function Tests}

The medical records of all 20 patients were reviewed to determine serum albumin levels, total bilirubin levels, international normalized ratios (INRs), and platelet counts from samples acquired within one week before or after MRI.ICG clearance tests were examined within two weeks before or after MRI. A $0.5 \mathrm{mg} / \mathrm{kg}$ ICG dose was administered intravenously and venous blood was sampled in which $5 \mathrm{ml}$ before and at 5,10 , and $15 \mathrm{~min}$ after ICG administration [17]. ICG-K was calculated by a linear regression analysis of plasma ICG concentrations. ICG-Krem was defined as preoperative ICG-K $\times$ FRL parenchymal volume/total liver parenchymal volume [1] [2]. The ICG-Krem threshold required for safe liver resection was $>0.05$ [3]. Twenty patients who had PHLF were evaluated after postoperative day five to determine INRs.

\subsection{Postoperative Clinical Outcomes}

PHLF was evaluated after postoperative day five using increased INRs that were defined according to the 
ISGLS. In this grading system, PHLF is characterized by increased INRs and concomitant hyperbilirubinemia on or after postoperative day five. The severity of PHLF is graded according to its impact on clinical management. Grade A PHLF requires no change in the patient's clinical management. The clinical management of patients with grade B PHLF deviates from the regular course but does not require invasive therapy. Grade C PHLF defines the need for invasive treatment. We made preoperative PHLF predictions according to the HLF-ICGKrem values.

\subsection{MRI}

All patients underwent MRI using a Signa HDxt 3.0Tclinical scanner with a superconducting magnet (GE Healthcare, Milwaukee, WI, USA) and an 8-channel phased-array coil. Dynamic images using fat-suppressed T1-weighted gradient-echo images with a 3-dimensional acquisition sequence (liver acquisition with volume acceleration) were acquired before and at $35 \mathrm{~s}, 90 \mathrm{~s}, 180 \mathrm{~s}$, and $20 \mathrm{~min}$ after the administration of gadoxetic acid disodium $(0.1 \mathrm{~mL} / \mathrm{kg}$ body weight). The gadoxetic acid disodium was administered intravenously as a bolus at a rate of $1 \mathrm{~mL} / \mathrm{s}$ through an intravenous cubital line that was flushed with $40 \mathrm{~mL}$ of saline via a power injector (Dual Shot GX, Nemotokyorindo, Tokyo, Japan). Images were acquired in the transverse plane at a section thickness of $3.8 \mathrm{~mm}$ (zero-fill interpolation: zip, 2). The parameters were as follows: repetition time/echo time, 3.1/1.4 s; flip angle, $12^{\circ}$; number of signals acquired, 1; field of view, $38 \mathrm{~cm}$; matrix, $256 \times 224$ (512 zip); and acquisition time, $20 \mathrm{~s}$.

\subsection{MRI-Based Liver Function Indices}

MRI-based liver function indices were the FRL-hepatocellular uptake index (rHUI), RLE, FRL-RLE (rRLE), coefficient variation of RLE [Cv(RLE)], coefficient variation of rRLE [Cv(rRLE)], and ICG-Krem corrected by HLF (HLF-ICG-Krem). In 20 patients who had PHLF, we investigated the correlations after postoperative day five between increased INRs and rHUI, rRLE, Cv(rRLE), and HLF-ICG-Krem.

\section{6. rHUI Hepatobiliary Phase Images}

rHUI values were defined on hepatobiliary phase images as follows:

$$
\mathrm{rHUI}=\mathrm{rV}_{\mathrm{L}}\left[\left(\mathrm{rL}_{20} / \mathrm{S}_{20}\right)-1\right]
$$

where $\mathrm{rV}_{\mathrm{L}}$ is the volume of the FRL. $\mathrm{rL}_{20}$ and $\mathrm{S}_{20}$ are the signal intensities of FRL, and spleen in the hepatobiliary phase, respectively.

\subsection{RLE Hepatobiliary Phase Images}

Two sets of axial images were acquired from two types of liver images: precontrast enhancement images and hepatobiliary phase images. Hepatobiliary phase images were acquired 20 min after the intravenous administration of gadoxetic acid disodium. The RLE on hepatobiliary phase images were considered to primarily reflect hepatocellular enhancement effects. RLE values were defined as the signal gain percentage between the precontrast images and hepatobiliary phase images as follows:

$$
\operatorname{RLEvalues}(\%)=\left[\left(\mathrm{SI}_{\mathrm{H}}-\mathrm{SI}_{\mathrm{P}}\right) / \mathrm{SI}_{\mathrm{P}}\right] \times 100
$$

where $\mathrm{SI}_{\mathrm{H}}$ and $\mathrm{SI}_{\mathrm{P}}$ are the signal intensities in the hepatobiliary phase images and precontrast enhanced images, respectively. RLE images were constructed on the basis of RLE values at each pixel and calculated using equation (2). Therefore, the signal intensity of each pixel was representative of the corresponding RLE value in the RLE images, which were displayed according to the National Institutes of Health color look-up table.

HLF was evaluated using the $\mathrm{Cv}(\mathrm{RLE})$ and was defined as follows:

$$
\mathrm{Cv}(\mathrm{RLE})=(\text { standard deviation of RLEvalue/average value of RLEvalue })
$$

ICG-Krem in consideration of HLF (HLF-ICG-Krem) was defined as follows:

$$
\text { HLF-ICG-Krem = ICG-Krem }[\mathrm{Cv}(\mathrm{RLE}) / \mathrm{Cv}(\mathrm{rRLE})]
$$


Correlations were evaluated between rHUI, rRLE, Cv(rRLE), HLF-ICG-Krem, and INRs after postoperative day five.

RLE values and liver parenchymal volumes were measured by two radiologists (Y.S. and S.M., with 18 and three years of experience reading MR images, respectively) and a PhD researcher (S.M., with 31 years of experience reading MR images). Region of interest (ROI) and volume of interest (VOI) acquisitions were performed using OsiriX (ver. 2.7.5) medical imaging software (http://www.osirix-viewer.com). In the whole liver and FRL, hepatobiliary phase images were used to define ROIs and VOIs for the tumor-free liver parenchyma by freehand contouring. The major vessels in the liver, such as the portal and hepatic veins, were excluded during ROI acquisition (Figure 1). ROIs were drawn on RLE images for RLE value analysis. We evaluated the whole liver in each image slice.

\subsection{Statistical Analyses}

Statistical analyses were performed using the software package SPSS for Macintosh (Version 16.0, SPSS Inc., Chicago, IL, USA). The Spearman correlation coefficient was used to evaluate correlations between rHUI, rRLE, Cv(rRLE), HLF-ICG-Krem, and INR values after postoperative day five. The Mann-Whitney test and Tukey's multiple comparison test were used to evaluate differences in rHUI and HLF-ICG-Krem values according to the classified PHLF grade. In the preoperative evaluation of PHLF, HLF-ICG-Krem values and PHLF grades were calculated and compared by receiver operating characteristic (ROC) curves. Two-tailed p values $<0.05$ were considered to be statistically significant.

\section{Results}

\subsection{Correlation between PHLF Grades and RLE Images}

Figure 2 shows RLE images from patients who did not have PHLF and patients with PHLF grade B that satisfied the ICG-Krem safety levels. Those patients without PHLF (upper row of Figure 2) exhibited higher RLE values. Both $\mathrm{Cv}(\mathrm{RLE})$ and $\mathrm{Cv}(\mathrm{rRLE})$ values were low. On the other hand, the PHLF grade B patients (bottom row of Figure 2) exhibited lower RLE values and both Cv(RLE) and Cv(rRLE) values were high.

\subsection{Correlation between INRs after Postoperative Day Five and HLF-ICG-Krem, Cv(rRLE), rREL, and rHUI}

Table 1 shows the correlations between INRs after postoperative day five and HLF-ICG-Krem, Cv(rRLE), rREL, and rHUI values. HLF-ICG-Krem and rREL values correlated with INRs after postoperative day five $(\mathrm{r}=$ -0.55 and $0.46, p=0.01$ and 0.04 , respectively). On the other hand, neither Cv(rRLE) nor rHUI was correlated with INRs after postoperative day five $(r=-0.03$ and 0.28 , respectively; $\mathrm{p}>0.05$ for both). Furthermore, HLFICG-Krem values $\leq 0.05$ detected two patients with higher INRs after postoperative day five (Figure 3).

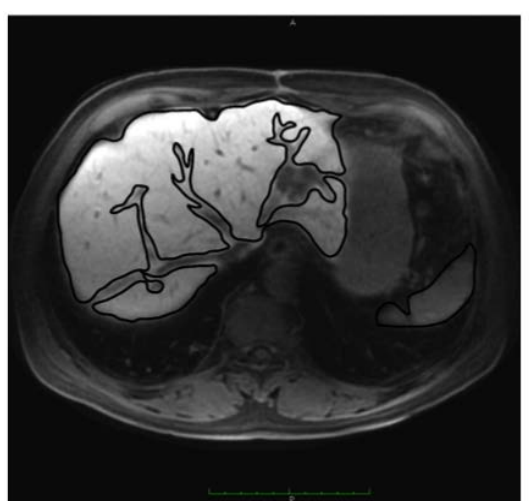

(a)

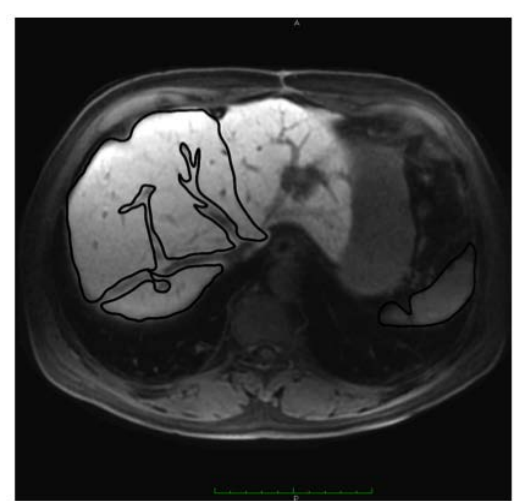

(b)

Figure 1. The defined region of interests for the whole liver and future remnant liver parenchyma, without liver tumors, in the hepatobiliary phase image. Black lines outline the liver and spleen. (a) whole liver; (b) future remnant liver. 


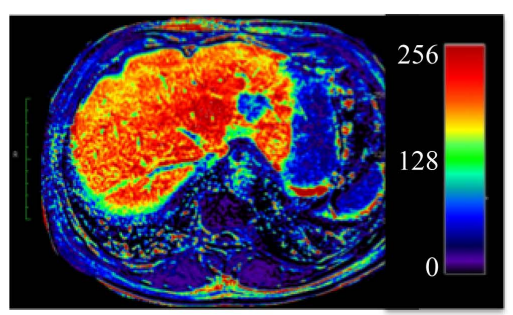

(a)

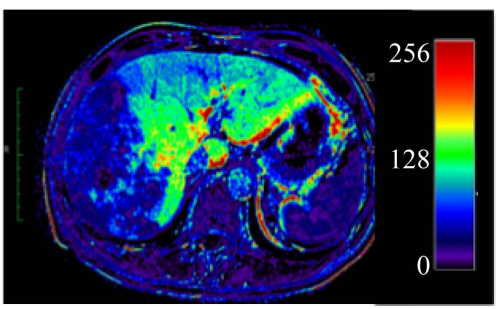

(d)

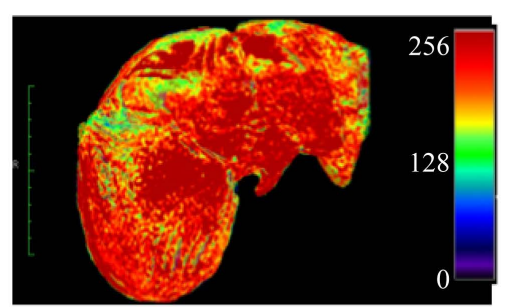

(b)

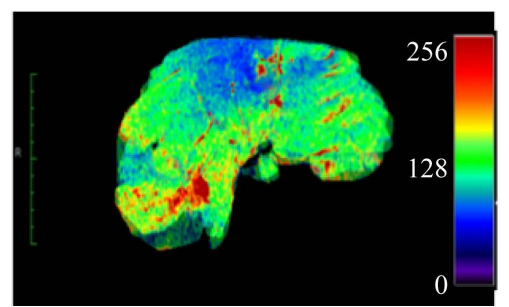

(e)

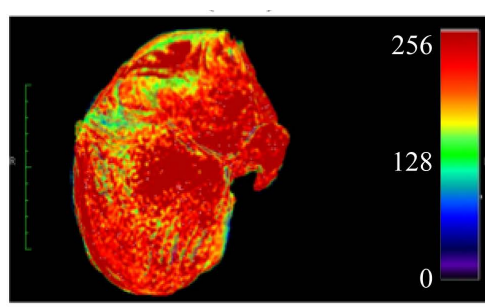

(c)

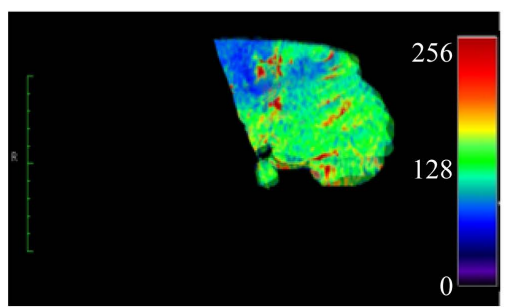

(f)

Figure 2. Relative liver enhancement (RLE) images in patients without posthepatectomy liver failure (PHLF) and those with PHLF grade B. The color bar denotes that red reflects high RLE values and black reflects low RLE values. (a) RLE images of a tumor center slice in patients without PHLF. (b) Total liver volume image of RLE in patients without PHLF; total liver volume $=1266.1 \mathrm{~cm}^{2}$, coefficient variation RLE $[\mathrm{Cv}(\mathrm{RLE})]=16.3 \%$. (c) Future remnant liver (FRL) volume image of RLE in patients with PHLF grade B; FRL volume $=1016.7 \mathrm{~cm}^{2}$, coefficient variation remnant RLE $[\mathrm{Cv}(\mathrm{rRLE})]=18.0 \%$, indocyanine green clearance of future remnant liver $($ ICG-Krem) $=0.155$, heterogeneous liver function $($ HLF-ICG-Krem) $=$ 0.140. (d) RLE images of a tumor center slice in patients with PHLF grade B. (e) Total liver volume image of RLE in patients with PHLF grade B; total liver volume $=1521.1 \mathrm{~cm}^{2}, \mathrm{Cv}(\mathrm{RLE})=27.3 \%$. (f) FRL volume image of RLE in patients with PHLF grade B; FRL volume $=532.1 \mathrm{~m}^{2}, \mathrm{Cv}(\mathrm{rRLE})=38.5 \%$, ICG-Krem $=0.061$, HLF-ICG-Krem $=0.043$.

Table 1. Correlation of INR after postoperative five day and evaluation parameters of PHLF in Child-pugh A patients.

\begin{tabular}{cccc}
\hline & & INR after postoperative day 5 & P value \\
\hline Indices & $\mathrm{r}$ & $95 \%$ confidence interval & 0.01 \\
\hline HLF-ICG-Krem & -0.55 & -0.81 to -0.13 & 0.04 \\
rREL & 0.46 & -0.05 to 0.75 & 0.89 \\
Cv(rREL) & -0.03 & -0.48 to 0.43 & 0.24 \\
rHUI & 0.28 & -0.20 to 0.65 & \\
\hline
\end{tabular}

HLF-ICG-Krem: heterogeneous liver function ICG-Krem, rRLE: Future remnant liver RLE, Cv(rREL): Coefficient variation rRLE, rHUI: remnant hepatocellular uptake index, REL: Relative enhancement of the liver.

\subsection{Clinical Data and MRI-Based Liver Function Indices}

Table 2 summarizes the clinical data as well as the PHLF grades and MRI-based liver function indices. Of the 20 evaluated patients, four (20\%) had elevated INR values and serum bilirubin levels on postoperative day five and thus met the criteria for liver failure as proposed by the ISGLS. Of these four patients, two (50\%) did not require specific treatment and were classified as PHLF grade A, the other two (50\%) required noninvasive treatment and were classified as PHLF grade B.

Table 3 summarizes the significant differences in PHLF grades, HLF-ICG-Krem, and rRLE values. HLFICG-Krem values were significantly lower in patients with PHLF $(0.062 \pm 0.021)$ than in those without PHLF $(0.125 \pm 0.031, \mathrm{p}<0.05)$. HLF-ICG-Krem values were significantly lower in patients with PHLF grade B $(0.045$ $\pm 0.004)$ than in those without PHLF $(\mathrm{p}<0.05)$.

Table 4 summarizes the disorder probability of HLF-ICG-Krem in the Child-Pugh class A independent group. The sensitivity, specificity, efficiency, prevalence, positive predictive value, and negative predictive value using a threshold HLF-ICG-Krem value of 0.05 that determined the presence of PHLF were 50\%, 100\%, 0.8\%, 0.2\%, 
Table 2. Summary of the clinical data and MRI-based liver function indices.

\begin{tabular}{|c|c|c|c|c|c|c|c|c|c|c|c|c|c|c|c|c|c|}
\hline \begin{tabular}{l}
$\dot{0}$ \\
$Z$ \\
\multirow{U}{U}{} \\
$ن$
\end{tabular} & ஸ் & $\stackrel{\infty}{4}$ & 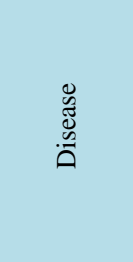 & 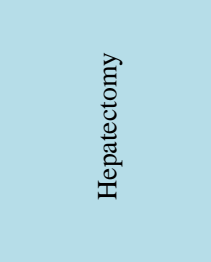 & 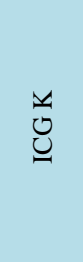 & 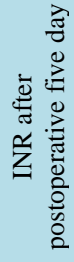 & 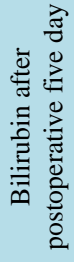 & 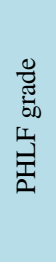 & 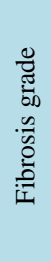 & $\sum_{i}^{+}$ & 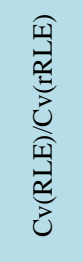 & 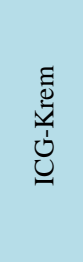 & 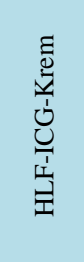 & 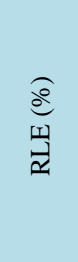 & 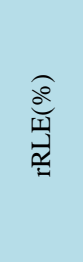 & 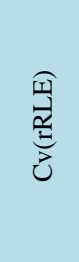 & 空 \\
\hline 1 & $\mathrm{~F}$ & 43 & HCC & $\begin{array}{c}\text { left } \\
\text { hepatectomy }\end{array}$ & 0.177 & 1.28 & 1.6 & A & F0 & 0.531 & 0.730 & 0.094 & 0.069 & 143.2 & 155.2 & 27.45 & 483.9 \\
\hline 2 & $\mathrm{M}$ & 72 & HCC & $\begin{array}{c}\text { Partial } \\
\text { hepatectomy }\end{array}$ & 0.120 & 1.28 & 2.2 & A & F0 & 0.737 & 1.000 & 0.088 & 0.088 & 150.8 & 151.7 & 21.95 & 698.1 \\
\hline 3 & $\mathrm{M}$ & 62 & HCC & $\begin{array}{c}\text { right } \\
\text { hepatectomy }\end{array}$ & 0.173 & 1.47 & 1.1 & B & $\mathrm{F} 4$ & 0.350 & 0.708 & 0.061 & 0.043 & 111.8 & 106.7 & 38.52 & 850.6 \\
\hline 4 & M & 65 & HCC & $\begin{array}{c}\text { central } \\
\text { bisegmentectomy }\end{array}$ & 0.118 & 1.98 & 3.6 & B & F0 & 0.439 & 0.926 & 0.052 & 0.048 & 128.8 & 138.9 & 51.33 & 373.8 \\
\hline 5 & $\mathrm{~F}$ & 69 & HCC & $\begin{array}{c}\text { medial left } \\
\text { segmentectomy }\end{array}$ & 0.172 & 1.20 & 0.4 & No & F3 & 0.605 & 0.978 & 0.104 & 0.102 & 164.3 & 164.8 & 21.97 & 403.7 \\
\hline 6 & $\mathrm{~F}$ & 59 & Metastasis & $\begin{array}{c}\text { lateral } \\
\text { segmentectomy }\end{array}$ & 0.196 & 1.00 & 0.8 & No & F0 & 0.698 & 0.762 & 0.137 & 0.104 & 147.3 & 149.5 & 33.04 & 569.2 \\
\hline 7 & $\mathrm{M}$ & 60 & Metastasis & $\begin{array}{c}\text { right } \\
\text { hepatectomy }\end{array}$ & 0.181 & 1.11 & 0.7 & No & F0 & 0.406 & 1.552 & 0.073 & 0.114 & 132.2 & 128.4 & 16.28 & 215.5 \\
\hline 8 & M & 64 & Metastasis & $\begin{array}{c}\text { partial } \\
\text { hepatectomy }\end{array}$ & 0.223 & 1.21 & 1.1 & No & F0 & 0.946 & 0.955 & 0.211 & 0.201 & 168.6 & 167 & 21.80 & 639.8 \\
\hline 9 & $\mathrm{M}$ & 63 & Metastasis & $\begin{array}{c}\text { lateral } \\
\text { segmentectomy }\end{array}$ & 0.193 & 1.18 & 0.8 & No & F0 & 0.803 & 0.904 & 0.155 & 0.140 & 198.9 & 203.2 & 18.01 & 1201.4 \\
\hline 10 & $\mathrm{M}$ & 82 & HCC & $\begin{array}{c}\text { partial } \\
\text { hepatectomy }\end{array}$ & 0.182 & 1.20 & 0.7 & No & $\mathrm{F} 1$ & 0.774 & 0.840 & 0.141 & 0.118 & 140.2 & 140 & 27.07 & 896.8 \\
\hline 11 & $\mathrm{M}$ & 60 & HCC & $\begin{array}{c}\text { medial left } \\
\text { segmentectomy }\end{array}$ & 0.178 & 1.20 & 1.2 & No & F0 & 0.777 & 0.973 & 0.138 & 0.135 & 172.3 & 177.1 & 23.26 & 1208.1 \\
\hline 12 & M & 68 & HCC & $\begin{array}{c}\text { right } \\
\text { hepatectomy }\end{array}$ & 0.191 & 1.27 & 0.7 & No & F0 & 0.361 & 0.941 & 0.069 & 0.065 & 153.3 & 153.4 & 20.86 & 298.2 \\
\hline 13 & M & 71 & Metastasis & $\begin{array}{c}\text { partial } \\
\text { hepatectomy }\end{array}$ & 0.149 & 1.10 & 0.5 & No & F0 & 0.880 & 0.816 & 0.131 & 0.107 & 98.8 & 100.1 & 31.37 & 130.4 \\
\hline 14 & $\mathrm{~F}$ & 46 & Metastasis & $\begin{array}{c}\text { partial } \\
\text { hepatectomy }\end{array}$ & 0.220 & 1.16 & 0.3 & No & F0 & 0.749 & 0.586 & 0.165 & 0.097 & 94.4 & 97 & 46.08 & 190.7 \\
\hline 15 & $\mathrm{M}$ & 69 & Metastasis & $\begin{array}{c}\text { partial } \\
\text { hepatectomy }\end{array}$ & 0.131 & 0.99 & 1.5 & No & F0 & 0.895 & 0.937 & 0.117 & 0.110 & 91.6 & 91.8 & 39.98 & 514.1 \\
\hline 16 & $\mathrm{~F}$ & 70 & Metastasis & $\begin{array}{c}\text { posterior } \\
\text { sectionectomy }\end{array}$ & 0.255 & 0.96 & 1.0 & No & F0 & 0.727 & 0.770 & 0.185 & 0.143 & 118.1 & 127.6 & 48.35 & 523.7 \\
\hline 17 & M & 75 & Metastasis & $\begin{array}{c}\text { partial } \\
\text { hepatectomy }\end{array}$ & 0.147 & 1.10 & 1.0 & No & F0 & 0.863 & 1.201 & 0.127 & 0.152 & 102.3 & 105.7 & 8.14 & 779.1 \\
\hline 18 & $\mathrm{~F}$ & 68 & Metastasis & $\begin{array}{c}\text { posterior } \\
\text { sectionectomy }\end{array}$ & 0.210 & 1.05 & 0.4 & No & F0 & 0.772 & 0.972 & 0.162 & 0.158 & 126 & 124.4 & 20.50 & 288.6 \\
\hline 19 & M & 60 & Metastasis & left hepatectomy & 0.148 & 1.16 & 1.0 & No & F0 & 0.749 & 1.176 & 0.111 & 0.130 & 80.8 & 84.2 & 29.57 & 208.2 \\
\hline 20 & M & 73 & Metastasis & $\begin{array}{c}\text { left lateral } \\
\text { segmentectomy }\end{array}$ & 0.135 & 1.08 & 0.6 & No & F0 & 0.832 & 1.100 & 0.112 & 0.124 & 113.2 & 113.9 & 21.60 & 63.0 \\
\hline
\end{tabular}

PHLF: posthepatectomy liver failure, VL: Volume of whole liver, rVL: Volume of whole future remnant liver, RLE: relative liver enhancement, rRLE: Future remnant liver RLE, Cv(REL): Coefficient variation RLE, Cv(rREL): Coefficient variation rRLE, HLF-ICG-Krem: heterogeneous liver function ICG-Krem, rHUI: remnant hepatocellular uptake index. 


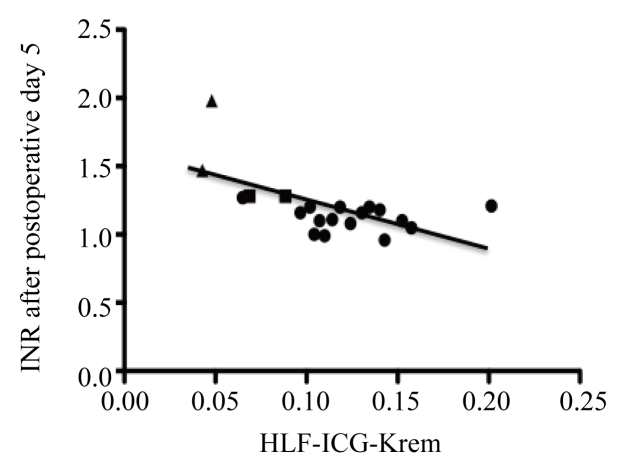

(a)

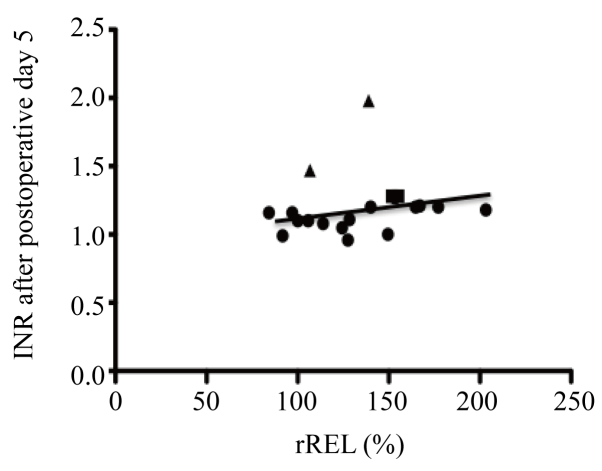

(b)

Figure 3. Correlations between international normalized ratios (INRs) after postoperative day five and heterogeneous liver function-indocyanine green clearance of future remnant liver (HLFICG-Krem), future remnant liver RLE (rRLE) values. The symbol $\bullet$ is No-posthepatectomy liver failure (PHLF), $\boldsymbol{n}$ is PHLF grade A, $\boldsymbol{\Delta}$ is PHLF grade B. (a) HLF-ICG-Krem ( $\mathrm{r}=-0.55, \mathrm{p}=$ 0.01). (b) $\operatorname{rRLE}(\mathrm{r}=0.46, \mathrm{p}=0.04)$.

Table 3. Summary of the significant differences of PHLF grades, HLF-ICG-Krem, and rRLE.

\begin{tabular}{ccccc} 
& $\mathrm{n}$ & HLF-ICG-Krem & rRLE \\
\hline No-PHLF & 16 & $0.125 \pm 0.031$ & & $133.0 \pm 34.1$ \\
PHLF & 4 & $0.062 \pm 0.021$ & $*$ & $138.1 \pm 22.1$ \\
PHLF grade A & 2 & $0.079 \pm 0.014$ & & $153.5 \pm 2.5$ \\
PHLF grade B & 2 & $0.045 \pm 0.004$ & $* *$ & $122.8 \pm 22.8$ \\
\hline
\end{tabular}

*: significant difference in No-PHLF and PHLF by Mann Whitney test; **: significant difference in No-PHLF and PHLF gred A, B by Tukey's Multiple comparison test; HLF-ICG-Krem: heterogeneous liver function, rRLE: relative liver enhancement of future remnant liver. PHLF: posthepatectomy liver failure.

Table 4. Disorder probability of HLF-ICG-Krem in the Child-Pugh class A independent group.

\begin{tabular}{ccc}
\hline & No-PHLF vs. PHLF & No-PHLF vs. PHLF B \\
\hline Cut off value & 0.05 & 0.05 \\
Sensitivity (\%) & $50(2 / 4)$ & $100(2 / 2)$ \\
Specificity (\%) & $100(16 / 16)$ & $100(18 / 18)$ \\
Efficiency & $0.8(16 / 20)$ & $1(20 / 20)$ \\
Prevalence & $0.2(4 / 20)$ & $0.1(2 / 18)$ \\
Positive predictive value (\%) & $100(2 / 2)$ & $100(2 / 2)$ \\
Negative predictive value (\%) & $89(16 / 18)$ & $100(18 / 18)$ \\
\hline
\end{tabular}

100\%, and 89\%, respectively. The sensitivity, specificity, efficiency, prevalence, positive predictive value, and negative predictive value using a threshold HLF-ICG-Krem value of 0.05 that determined the presence of PHLF grade B were $100 \%, 100 \%, 1 \%, 0.1 \%, 100 \%$, and $100 \%$, respectively.

\section{Discussion}

Liver function is usually evaluated using laboratory data obtained by blood sampling.ICG is one of the important test that comprehensively evaluates hepatic function, including uptake, metabolism, and excretion [18] [19]. Recent reports suggest that gadoxetic acid disodium can also be used as a tracer for liver function testing [5]-[7] [10]-[14]. It is feasible to analyse the contrast agent accumulation in the hepatobiliary phase, as has been described 
by Motosugi et al. and Yamada et al., who used a semi-quantitative approach relating the liver SI to the splenic SI [6] [7]. Yamada et al. reported that HUI and $\mathrm{V}_{\mathrm{S}}$ were the factors significantly correlated with the plasma disappearance rate of ICG [7].

Preoperative evaluations of FRL function are crucial for determining whether a patient can safely undergo liver resection. Numerous attempts have been made to define prognostic factors that would facilitate liver failure risk assessments [20]-[22]. Hoekstra et al., [20] evaluated several liver function tests to determine their abilities to predict liver failure. The authors concluded that "in addition to volumetry, quantitative liver function tests should be used to determine whether a safe resection can be performed". The ICG clearance test is by far the most widely administered dynamic liver function test [23]. According to Wibmer et al., [22] in patients without HCC, impaired liver parenchymal enhancement in the hepatobiliary phase of preoperative gadoxetic acidenhanced MRI was independently associated with a higher risk of liver failure after major liver resection. Liver volumetry is essential when evaluating patients who are candidates for partial liver resection because the postoperative treatment strongly depends on the liver remnant volume [24]. In Europe and America, patients with Child-Pugh class B and C scores are commonly not considered to be surgery adaptive. Furthermore, in patients with Child-Pugh class A scores and portal hypertension, an outside, adaptive hepatectomy was recommended [25]. In our study, we used the ICG-Krem value, which was calculated as the ratio of FRL volume to total liver volume, to estimate PHLF in the Child-Pugh class A. However, ICG-Krem does not consider HLF. We evaluated PHLF, which was undetectable in ICG-Krem, by HLF-ICG-Krem.

This study included both patients with metastatic liver tumors and those with HCC, and the liver excision methods included both major and partial liver resection. Both rRLE and HLF-ICG-Krem values correlated with INRs after postoperative day five. However, neither Cv(rRLE) nor rHUI was correlated with INRs after postoperative day five. This result suggests that rRLE and HLF-ICG-Krem are parameters that could be used to evaluate PHLF independently of the case or operation method.

HLF-ICG-Krem was significantly lower in patients with PHLF than in those without PHLF $(\mathrm{p}<0.05)$. When the HLF-ICG-Krem threshold value was set at 0.05, HLF-ICG-Krem detected two patients among four with PHLF, which was not detected by ICG-Krem. HLF-ICG-Krem was significantly lower in patients with PHLF grade B than in those without PHLF ( $<0.05$ ), at the $<0.05$ threshold value. The ability to predict which patients will develop PHLF grade B prior to surgery is clinically meaningful because the postoperative management of such patients deviates from the regular course. This study has some limitations. First, the study population included a small sample size, and second, the study population included few cases of PHLF grade A or B, and no cases of grade $\mathrm{C}$.

\section{Conclusion}

In summary, the HLF-ICG-Krem value incorporates HLF into the standard ICG-Krem value and thus provides a useful parameter that can evaluate PHLF more correctly. This MRI examination method also provides morphological and functional information that can be used to optimize the preoperative selection of patients who can safely undergo liver resection.

\section{Acknowledgements}

The authors thank Mamoru Sogami, MD, PhD for providing valuable advice and all the study participants for their cooperation.

\section{Funding}

This work was supported by JSPS KAKENHI (26461844).

\section{References}

[1] Kurumiya, Y., Nagino, M., Nozawa, K., et al. (2003) Biliary Bile Acid Concentration Is a Simple and Reliable Indicator for Liver Function after Hepatobiliary Resection for Biliary Cancer. Surgery, 133, 512-520. http://dx.doi.org/10.1067/msy.2003.142

[2] Uesaka, K., Nimura, Y. and Nagino, M. (1996) Changes in Hepatic Lobar Function after Right Portal Vein Embolization. An Appraisal by Biliary Indocyanine Green Excretion. Annals of Surgery, 223, 77-83.

http://dx.doi.org/10.1097/00000658-199601000-00011 
[3] Nagino, M., Kamiya, J., Nishio, H., Ebata, T., Arai, T. and Nimura, Y. (2006) Two Hundred Forty Consecutive Portal vein Embolizations before Extended Hepatectomy for Biliary Cancer: Surgical Outcome and Long-Term Follow-Up. Annals of Surgery, 243, 364-372. http://dx.doi.org/10.1097/01.sla.0000201482.11876.14

[4] Albers, I., Hartmann, H., Bircher, J. and Creutzfeldt, W. (1989) Superiority of the Child-Pugh Classification to Quantitative Liver Function Tests for Assessing Prognosis of Liver Cirrhosis. Scandinavian Journal of Gastroenterology, 24, 269-276. http://dx.doi.org/10.3109/00365528909093045

[5] Motosugi, U., Ichikawa, T., Sou, H., et al. (2009) Liver Parenchymal Enhancement of Hepatocyte-Phase Images in Gd-EOB-DTPA-Enhanced MR Imaging: Which Biological Markers of the Liver Function Affect the Enhancement? Journal of Magnetic Resonance Imaging, 30, 1042-1046. http://dx.doi.org/10.1002/jmri.21956

[6] Motosugi, U., Ichikawa, T., Tominaga, L., et al. (2009) Delay before the Hepatocyte Phase of Gd-EOB-DTPAEnhanced MR Imaging: Is It Possible to Shorten the Examination Time? European Radiology, 19, 2623-2629. http://dx.doi.org/10.1007/s00330-009-1467-6

[7] Yamada, A., Hara, T., Li, F., et al. (2011) Quantitative Evaluation of Liver Function with Use of Gadoxetate Disodium-Enhanced MR Imaging. Radiology, 260, 727-733. http://dx.doi.org/10.1148/radiol.11100586

[8] Ohwada, S., Kawate, S., Hamada, K., et al. (2006) Perioperative Real-Time Monitoring of Indocyanine Green Clearance by Pulse Spectrophotometry Predicts Remnant Liver Functional Reserve in Resection of Hepatocellular Carcinoma. British Journal of Surgery, 93, 339-346. http://dx.doi.org/10.1002/bjs.5258

[9] Kwon, A.H., Ha-Kawa, S.K., Uetsuji, S., Inoue, T., Matsui, Y. and Kamiyama, Y. (1997) Preoperative Determination of the Surgical Procedure for Hepatectomy Using Technetium-99m-Galactosyl Human Serum Albumin (99mTc-GSA) Liver Scintigraphy. Hepatology, 25, 426-429. http://dx.doi.org/10.1002/hep.510250228

[10] Tajima, T., Takao, H., Akai, H., et al. (2010) Relationship between Liver Function and Liver Signal Intensity in Hepatobiliary Phase of Gadolinium Ethoxybenzyl Diethylenetriamine Pentaacetic Acid-Enhanced Magnetic Resonance Imaging. Journal of Computer Assisted Tomography, 34, 362-366. http://dx.doi.org/10.1097/RCT.0b013e3181cd3304

[11] Kim, H.Y., Choi, J.Y., Park, C.H., et al. (2013) Clinical Factors Predictive of Insufficient Liver Enhancement on the Hepatocyte-Phase of Gd-EOB-DTPA-Enhanced Magnetic Resonance Imaging in Patients with Liver Cirrhosis. Journal of Gastroenterology, 48, 1180-1187. http://dx.doi.org/10.1007/s00535-012-0740-7

[12] Yoneyama, T., Fukukura, Y., Kamimura, K., et al. (2014) Efficacy of Liver Parenchymal Enhancement and Liver Volume to Standard Liver Volume Ratio on Gd-EOB-DTPA-Enhanced MRI for Estimation of Liver Function. European Radiology, 24, 857-865. http://dx.doi.org/10.1007/s00330-013-3086-5

[13] Matsushima, S., Sato, Y., Yamaura, H., et al. (2014) Visualization of Liver Uptake Function Using the Uptake ContrastEnhanced Ratio in Hepatobiliary Phase Imaging. Magnetic Resonance Imaging, 32, 654-659. http://dx.doi.org/10.1016/j.mri.2014.02.017

[14] Kukuk, G.M., Schaefer, S.G., Fimmers, R., et al. (2014) Hepatobiliary Magnetic Resonance Imaging in Patients with Liver Disease: Correlation of Liver Enhancement with Biochemical Liver Function Tests. European Radiology, 24, 2482-2490. http://dx.doi.org/10.1007/s00330-014-3291-x

[15] Rahbari, N.N., Garden, O.J., Padbury, R., et al. (2011) Posthepatectomy Liver Failure: A Definition and Grading by the International Study Group of Liver Surgery (ISGLS). Surgery, 149, 713-724. http://dx.doi.org/10.1016/j.surg.2010.10.001

[16] Matsumata, T., Kanematsu, T., Yoshida, Y., Furuta, T., Yanaga, K. and Sugimachi, K. (1987) The Indocyanine Green Test Enables Prediction of Postoperative Complications after Hepatic Resection. World Journal of Surgery, 11, 678681. http://dx.doi.org/10.1007/BF01655848

[17] Imamura, H., Sano, K., Sugawara, Y., Kokudo, N. and Makuuchi, M. (2005) Assessment of Hepatic Reserve for Indication of Hepatic Resection: Decision Tree Incorporating Indocyanine Green Test. Journal of Hepato-Biliary-Pancreatic Surgery, 12, 16-22. http://dx.doi.org/10.1007/s00534-004-0965-9

[18] Matsumata, T., Kanematsu, T., Yoshida, Y., Furuta, T., Yanaga, K. and Sugimachi, K. (1987) The Indocyanine Green Test Enables Prediction of Postoperative Complications after Hepatic Resection. World Journal of Surgery, 11, 678681. http://dx.doi.org/10.1007/BF01655848

[19] Lau, H., Man, K., Fan, S.T., Yu, W.C., Lo, C.M. and Wong, J. (1997) Evaluation of Preoperative Hepatic Function in Patients with Hepatocellular Carcinoma Undergoing Hepatectomy. British Journal of Surgery, 84, 1255-1259. http://dx.doi.org/10.1002/bjs.1800840917

[20] Hoekstra, L.T., de Graaf, W., Nibourg, G.A., et al. (2013) Physiological and Biochemical Basis of Clinical Liver Function Tests: A Review. Annals of Surgery, 257, 27-36. http://dx.doi.org/10.1097/SLA.0b013e31825d5d47

[21] Cho, S.H., Kang, U.R., Kim, J.D., Han, Y.S. and Choi, D.L. (2011) The Value of Gadoxetate Disodium Enhanced MR Imaging for Predicting Posthepatectomy Liver Failure after Major Hepatic Resection: A Preliminary Study. European Journal of Radiology, 80, e195-e200. http://dx.doi.org/10.1016/j.ejrad.2011.08.008 
[22] Wibmer, A., Prusa, A.M., Nolz, R., Gruenberger, T., Schindl, M. and Ba-Ssalamah, A. (2013) Liver Failure after Major Liver Resection: Risk Assessment by Using Preoperative Gadoxetic Acid-Enhanced 3-T MR Imaging. Radiology, 269, 777-786. http://dx.doi.org/10.1148/radiol.13130210

[23] Breitenstein, S., Apestegui, C., Petrowsky, H. and Clavien, P.A. (2009) "State of the Art” in Liver Resection and Living Donor Liver Transplantation: A Worldwide Survey of 100 Liver Centers. World Journal of Surgery, 33, 797-803. http://dx.doi.org/10.1007/s00268-008-9878-0

[24] Guglielmi, A., Ruzzenente, A., Conci, S., Valdegamberi, A. and Iacono, C. (2012) How Much Remnant Is Enough in Liver Resection? Digestive Surgery, 29, 6-17. http://dx.doi.org/10.1159/000335713

[25] Bruix, J. and Sherman, M. (2011) American Association for the Study of Liver Diseases. Management of Hepatocellular Carcinoma: An Update. Hepatology, 53, 1020-1022. http://dx.doi.org/10.1002/hep.24199

\section{Submit or recommend next manuscript to SCIRP and we will provide best service for you:}

Accepting pre-submission inquiries through Email, Facebook, Linkedin, Twitter, etc A wide selection of journals (inclusive of 9 subjects, more than 200 journals)

Providing a 24-hour high-quality service

User-friendly online submission system

Fair and swift peer-review system

Efficient typesetting and proofreading procedure

Display of the result of downloads and visits, as well as the number of cited articles

Maximum dissemination of your research work

Submit your manuscript at: http://papersubmission.scirp.org/ 